\title{
Computing Binding Energies of Interstellar Molecules by Semiempirical Quantum Methods: Comparison Between DFT and GFN2 on Crystalline Ice
}

\author{
Aurèle Germain $^{1(\bowtie)}(\mathbb{0})$, Marta Corno ${ }^{1,2}\left(\mathbb{0}\right.$, and Piero Ugliengo ${ }^{1,2}(\mathbb{0}$ \\ 1 Dipartimento di Chimica, Università degli Studi di Torino, \\ via P. Giuria 7, 10125 Torino, Italy \\ aureleroger.germain@unito.it \\ 2 Nanostructured Interfaces and Surfaces (NIS) Centre, Università degli Studi di \\ Torino, via P. Giuria 7, 10125 Torino, Italy
}

\begin{abstract}
Interstellar Grains (IGs) spread in the Interstellar Medium (ISM) host a multitude of chemical reactions that could lead to the production of interstellar Complex Organic Molecules (iCOMs), relevant in the context of prebiotic chemistry. These IGs are composed of a silicatebased core covered by several layers of amorphous water ice, known as a grain mantle. Molecules from the ISM gas-phase can be adsorbed at the grain surfaces, diffuse and react to give iCOMs and ultimately desorbed back to the gas phase. Thus, the study of the Binding Energy (BE) of these molecules at the water ice grain surface is important to understand the molecular composition of the ISM and its evolution in time. In this paper, we propose to use a recently developed semiempirical quantum approach, named GFN-xTB, and more precisely the GFN2 method, to compute the $\mathrm{BE}$ of several molecular species at the crystalline water ice slab model. This method is very cheap in term of computing power and time and was already showed in a previous work to be very accurate with small water clusters. To support our proposition, we decided to use, as a benchmark, the recent work published by some of us in which a crystalline model of proton-ordered water ice (P-ice) was adopted to predict the BEs of 21 molecules relevant in the ISM. The relatively good results obtained confirm GFN2 as the method of choice to model adsorption processes occurring at the icy grains in the ISM. The only notable exception was for the $\mathrm{CO}$ molecule, in which both structure and BE are badly predicted by GFN2, a real pity due to the relevance of $\mathrm{CO}$ in astrochemistry.
\end{abstract}

Keywords: Interstellar medium - Interstellar icy grains $\cdot$ Complexes organic molecules $\cdot$ Semiempirical quantum methods $\cdot$ GFN2

\section{Introduction}

Astronomical observations tell us that the Interstellar Medium (ISM) is rich in molecules that may become relevant in the prebiotic context.

(c) The Author(s) 2021

O. Gervasi et al. (Eds.): ICCSA 2021, LNCS 12953, pp. 632-645, 2021.

https://doi.org/10.1007/978-3-030-86976-2_43 
These molecules can be produced by diverse chemical processes inside the gas phase of the ISM, but some molecules like $\mathrm{H}_{2}$ (the most abundant molecule in the ISM $[15,17])$ cannot be formed by simple gas-phase process [15, 17]. However, the presence inside the ISM of micron-sized particles called Interstellar Grains (IGs), made of a silicate core covered by several layers of amorphous ice mantles $[3,12,18]$, are the place where $\mathrm{H}$ atoms get adsorbed and the excess energy dispersed into the grain when $\mathrm{H}_{2}$ is finally formed. Indeed, these IGs host chemical reactions producing interstellar Organic Molecules (iCOMs) by absorbing the excess of energy due to the bond formation of the iCOMs [13], while also acting, in specific cases, as a catalyst by reducing the activation energy $[7,14]$. Thus, chemical reactions at the surfaces of IGs could explain the presence in the ISM of molecules not feasible through gas phase processes.

The information about binding energies (BEs) of interstellar molecules on the grain is, therefore, essential for understanding the adsorption, movement, and desorption of iCOMs. All this is essential in order to understand the amount and distribution of iCOM in the gas-phase, as the BE controls both the diffusivity and the velocity of desorption from the grain towards the gas-phase where further processing of the iCOM will occur. Numerical models are, indeed, based on these data to provide the chemical composition of the ISM.

The BEs can be worked out in terrestrial laboratory by temperature programmed desorption (TPD), but the extreme conditions of the ISM (low temperature, low pressure, and very long time scale) hinders the real possibility to mimic the actual interstellar conditions. Quantum computation can be adopted as a useful complementary tool to the experimental data, provided that proper comparison between TPD extracted BE and those computed by quantum mechanical methods are properly made [8].

\section{Methodology}

\subsection{Computational Method}

In this work we aim at benchmarking a recently developed semiempirical quantum mechanical method (SQM) called GFN-xTB and more precisely the GFN2 method [1,2]. This method is being developed by the Grimme's group at the Bonn University and can be almost three orders of magnitude faster than conventional DFT methods; it can be used efficiently on simple desk computers and, indeed, every result showed in this paper was computed using a laptop computer equipped with an Intel i5 CPU and 8 GB of RAM.

We chose to use GFN-xTB not only because it is inexpensive in time and computational power, but also because it is particularly well suited for the system we want to study (IG made mostly of water ice), owning to its excellent performance in treating systems dominated by non-covalent interactions (H-bonds included), as it is the case of $\mathrm{iCOMs}$ adsorbed on water ice.

In a previous work [10], we showed that the method was accurate to deal with small water clusters for both energy and structures; here, we extended 
the applicability of the GFN2 method to model the interaction between a large number of relevant iCOMs adsorbed on a crystalline model of water ice. Our target is to establish whether GFN2 can compete with more robust and accurate DFT methods, as recently adopted by some of us [8], to model realistic and amorphous water clusters close to the real grains compared to the crystalline ice adopted here.

\subsection{Computational Dataset}

The set of data used to benchmark the GFN2 method for the computation of BEs was taken from Ferrero et al. 2020 work [8].

In that work Ferrero et al. [8] computed the BEs of 21 interstellar molecules, four of which were radicals. The BEs were computed using DFT/A-VTZ* method (M06-2X for radicals and B3LYP-D3 for closed shell ones).

In contrast to other computational chemistry work on BEs which use grain models composed of a few water molecules [6,16], Ferrero et al. [8] used a crystalline (010) slab model of proton-ordered ice (P-ice) [4] to simulate the grain structure, within the paradigm of the periodic boundary conditions. This crystalline model does not properly represent IGs, known to be amorphous. Nonetheless, the periodic boundaries used in the work by Ferrero et al. [8] indicated that the hydrogen bond cooperativity, too often underestimated in small water clusters mimicking the icy grains, is accurately represented.

Ferrero et al. [8] also adopted an amorphous periodic water model to study the BEs. This amorphous water slab is closer to the expected structure of IGs compared to a crystalline structure, but is not taken into account here as it is too difficult to simulate by a finite cluster (vide infra) as we did for the crystalline ice model.

\subsection{Crystalline Slab Model}

We cannot perform a one by one comparison with the Ferrero et al. [8] results as the periodic boundary condition (PBC) implementation in GFN-xTB does not include the GFN2 hamiltonian. Furthermore, the PBC coding in the xTB program is still under development. Therefore, we prefer to use a large molecular cube envisaging 84 water molecules (see Fig. 1) cut out from the periodic crystalline slab by Ferrero et al. [8].

This slab ensures that the dangling hydrogen $(\mathrm{dH})$ and oxygen $(\mathrm{dO})$ shown in Fig. 1, and acting as a binding sites, are properly embedded within surrounding layers of water molecules.

To simulate geometric constraints due to the long-range effects of the periodic boundaries applied to the $\mathrm{a}$ and $\mathrm{b}$ axis, we fixed the $\mathrm{H}$ atoms of the water molecules at the border of the cluster to the periodic conditions, while leaving free to move the $\mathrm{H}$ atoms within the core of the cluster. This ensures that the dangling hydrogen $\mathrm{dH}$, used in partnership with the dangling oxygen $\mathrm{dO}$ as a binding site for the different chemical species, is unconstrained and free to move. 

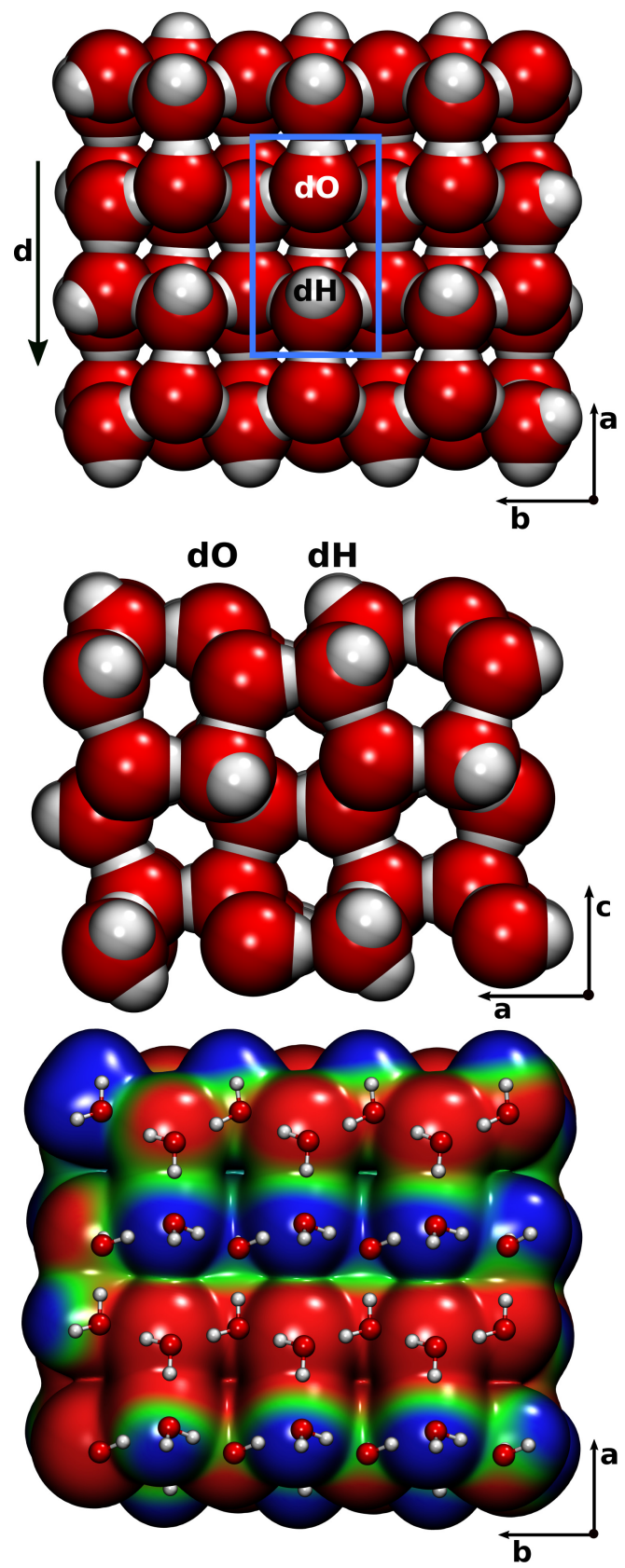

Fig. 1. Slab cut from the proton-ordered P-ice crystal used by Ferrero et al. 2020 [8]. Top: Slab seen from the ab plane with the $\mathrm{dO}$ and $\mathrm{dH}$ binding sites indicated inside the blue rectangle. The net dipole moment is indicated by a black arrow. Center: Slab seen from the ac plane. Bottom: Electrostatic potential mapped on the electron density of the slab. Negative regions are in red and positive regions are in blue. (Color figure online) 
These constrains will also avoid the collapse of the structure during geometry optimisation due to the missing water molecules at the frontiers of the cluster.

\subsection{Binding Energies and Role of the ZPE Correction}

For each chemical species to be studied we performed a GFN2 optimisation using the "extreme" level of optimisation of GFN-xTB. For each molecule, three optimisations were performed, one for the molecule alone, one for the slab alone, and one for both the slab and the molecule in interaction. The starting positions of the molecules above the binding site were as similar as possible to that adopted by Ferrero et al. 2020 [8] and optimised using GFN2 to check if the GFN-xTB positions after optimisation matched that of the DFT/A-VTZ* calculations. If the final position were significantly different, the starting position of the molecule was changed by trying to reproduce the final position of Ferrero et al. [8] instead of the initial one. The computation was then restarted (in the remainder of this article results for which the final position has been used will be followed by the suffix "-end". For example $\mathrm{N}_{2}$ indicates results using the starting position and $\mathrm{N}_{2}$-end indicates results using the final position). This way, we were able to check if the method could maintain this position as a stable one.

The BEs of these chemical species were computed as follow:

$$
B E=E_{m o l}+E_{\text {slab }}-E_{m o l+s l a b}
$$

Where $E_{m o l}, E_{s l a b}$, and $E_{\text {slab+mol }}$ are the GFN2 energies after optimisation of the molecule, the 84 water molecules slab with the constrains applied, and the molecule interacting with the slab, respectively.

To confirm that the optimised geometries are minima on the potential energy surface we computed the harmonic vibrational frequencies and checked that all frequencies were real. In case of imaginary frequencies, xTB provides a perturbed geometry which, after optimisation and further vibrational frequency calculation, may end up in a minimum.

From the set of frequencies, also the zero point energy $(Z P E)$ is provided for each component of the $\mathrm{BE}$ as:

$$
\triangle Z P E=Z P E_{\text {slab+mol }}-Z P E_{m o l}-Z P E_{\text {slab }}
$$

We can then obtain the zero point energy corrected binding energies $\mathrm{BE}(0)$ by subtracting $\triangle Z P E$ to the original $\mathrm{BE}$ :

$$
B E(0)=B E-\triangle Z P E
$$

In general, the $\triangle Z P E$ is a positive quantity, therefore the $B E(0)$ is always smaller than the uncorrected one. 


\section{Results and Discussion}

The adoption of a finite water cluster to mimic the PBC is not drawback-free. The first one, is the presence of a dipole moment of 14.4 Debye in the a-b plane of the slab and directed along the a cell axis (see Fig. 1). This dipole moment is exactly cancelled out when rigorous PBC are adopted, as in the work by Ferrero et al. [8]. In our case it could alter the behaviour of the most dipolar molecules of the set. The second one, is the fact that even if we try to mimic the long range effects imposed by the periodic boundaries conditions by applying constrains on the slab, this does not include the infinite hydrogen bond cooperativity of the real crystalline material. As a result, the final positions of these molecules could also be impacted.

Table 1. GFN2 BE, zero point energy corrected $\mathrm{BE}(0)$ and $\triangle \mathrm{ZPE}$ values (in $\mathrm{kJ} / \mathrm{mol}$ ) for all considered species. For $\mathrm{H}_{2} \mathrm{CO}$ and $\mathrm{HCONH}_{2}$ "SC1" and "SC2" refer to different initial positions used for the molecules on the binding sites by Ferrero et al. 2020 [8].

\begin{tabular}{|c|c|c|c|c|c|c|}
\hline \multirow{2}{*}{ Species } & \multicolumn{2}{|l|}{$\mathrm{BE}$} & \multicolumn{2}{|l|}{$\mathrm{BE}(0)$} & \multicolumn{2}{|l|}{$\triangle \mathrm{ZPE}$} \\
\hline & GFN2 & DFT & GFN2 & DFT & GFN2 & DFT \\
\hline $\mathrm{H}_{2}$ & 8.47 & 9.90 & 3.48 & 2.40 & 5.0 & 7.5 \\
\hline $\mathrm{O}_{2}$ & 40.6 & 8.50 & 47.8 & 6.80 & -7.1 & 1.7 \\
\hline $\mathrm{N}_{2}$ & 16.8 & 13.0 & 12.6 & 10.4 & 4.2 & 2.6 \\
\hline $\mathrm{N}_{2}$-end & 15.6 & 13.0 & 13.1 & 10.4 & 2.5 & 2.6 \\
\hline $\mathrm{CH}_{4}$ & 21.9 & 14.0 & 16.2 & 11.2 & 5.8 & 2.8 \\
\hline $\mathrm{CO}$ & 39.6 & 19.6 & 35.5 & 15.7 & 4.1 & 3.9 \\
\hline $\mathrm{CO}_{2}$ & 32.7 & 28.6 & 29.4 & 25.6 & 3.3 & 3.0 \\
\hline OCS & 27.8 & 28.9 & 25.4 & 26.5 & 2.4 & 2.4 \\
\hline $\mathrm{HCl}$ & 66.4 & 54.1 & 58.2 & 47.4 & 8.2 & 6.7 \\
\hline $\mathrm{HCN}$ & 35.5 & 42.6 & 31.2 & 41.6 & 4.2 & 1.0 \\
\hline $\mathrm{H}_{2} \mathrm{O}$ & 70.0 & 70.1 & 58.1 & 56.3 & 11.9 & 13.8 \\
\hline $\mathrm{H}_{2} \mathrm{~S}$ & 44.2 & 47.2 & 35.0 & 38.2 & 9.2 & 9.0 \\
\hline $\mathrm{NH}_{3}$ & 59.6 & 61.3 & 50.0 & 50.1 & 9.6 & 11.2 \\
\hline $\mathrm{CH}_{3} \mathrm{CN}$ & 52.9 & 62.8 & 47.0 & 56.6 & 6.0 & 6.2 \\
\hline $\mathrm{CH}_{3} \mathrm{OH}$ & 70.7 & 72.2 & 62.7 & 62.6 & 8.0 & 9.6 \\
\hline $\mathrm{H}_{2} \mathrm{CO}-\mathrm{SC} 1$ & 53.0 & 48.8 & 42.5 & 39.6 & 10.5 & 9.2 \\
\hline $\mathrm{H}_{2} \mathrm{CO}-\mathrm{SC} 2$ & 43.8 & 53.0 & 36.0 & 43.9 & 7.8 & 9.1 \\
\hline $\mathrm{HCONH}_{2}-\mathrm{SC} 1$ & 84.0 & 79.9 & 75.1 & 69.5 & 8.9 & 10.4 \\
\hline $\mathrm{HCONH}_{2}-\mathrm{SC} 2$ & 80.8 & 83.8 & 71.2 & 71.3 & 9.6 & 12.5 \\
\hline $\mathrm{HCOOH}$ & 86.4 & 78.5 & 81.5 & 71.7 & 4.9 & 6.8 \\
\hline $\mathrm{OH}$ & 115 & 54.4 & 127 & 43.3 & -11.8 & 11.1 \\
\hline $\mathrm{HCO}$ & 38.5 & 28.9 & 31.4 & 20.2 & 7.1 & 8.7 \\
\hline $\mathrm{CH}_{3}$ & 35.8 & 21.3 & 29.8 & 14.3 & 6.0 & 7.0 \\
\hline $\mathrm{CH}_{3}$-end & 23.8 & 21.3 & 17.4 & 14.3 & 6.4 & 7.0 \\
\hline
\end{tabular}

The binding energy values are given in Table 1. 
GFN2 results are in good agreement with the DFT ones except for some unusual cases, mainly: $\mathrm{CH}_{3}, \mathrm{CO}, \mathrm{HCO}, \mathrm{OH}$, and $\mathrm{O}_{2}$. All of them, except $\mathrm{CO}$, are radicals (the ground state of $\mathrm{O}_{2}$ is a triplet) making them highly reactive. Thus, they demand a level of theory that GFN2 cannot provide.
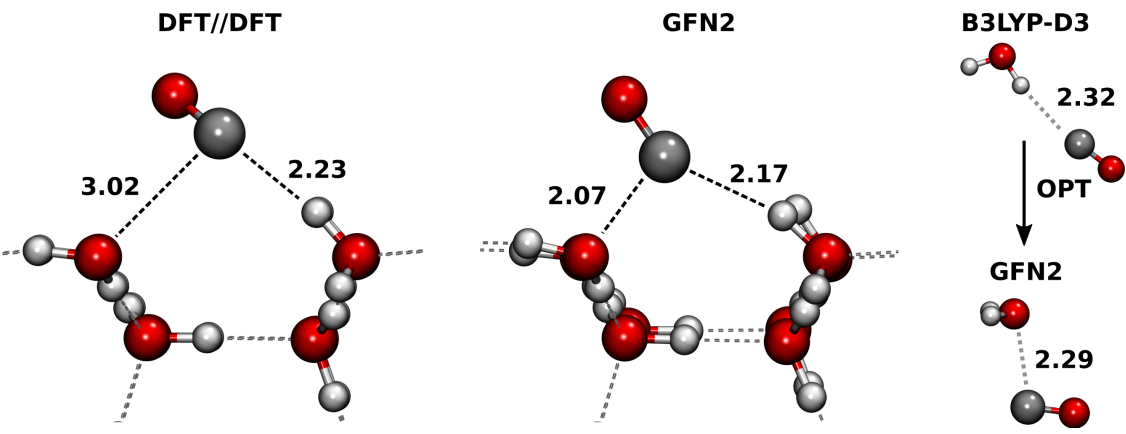

Fig. 2. Left: DFT//DFT results for CO. From Ferrero et al. 2020 [8]. Centre: GFN2 results for CO. Right: $\mathrm{H}_{2} \mathrm{O}-\mathrm{CO}$ dimer with the starting geometry from B3LYPD3/aug-cc-pVTZ on top and the GFN2 optimised geometry at the bottom. Dotted lines were added to emphasise the $\mathrm{H}-\mathrm{C}$ and $\mathrm{C}--\mathrm{O}$ interactions.

The particularly intriguing electron configuration of $\mathrm{CO}$ gives to the molecule a very weak dipole moment (with the negative pole located at the $\mathrm{C}$ atom) and a large quadrupole. The experimental dipole moment is $0.11 \mathrm{D}$ [11], with carbon atom carrying the negative end despite being less electronegative than oxygen (see Ref. [9]). GFN2 overestimates the dipole moment (0.615 D) and swaps the charges $(+0.0049 e$ on carbon) with respect to accurate calculations. This affect the results as, usually, CO interacts preferentially from the C-end when involved in H-bond interactions (see Fig. 2 Left) or with net charges like $\mathrm{Na}^{+}$. Unfortunately, GFN2 is unable to treat such a delicate bonding situation for CO interacting with the grain (see Fig. 2 Centre). Even when $\mathrm{CO}$ is put in interaction with a single water molecule, the optimised structure differs dramatically from the optimum B3LYP-D3/aug-cc-pvTZ one taken as internal reference, due to the bad description of the electronic structure of CO (see Fig. 2 Right). This is very unfortunate, owning to the general good performance of GFN2, and due to the extreme relevance of $\mathrm{CO}$ in the astrochemical context (the second most abundant gas-phase molecule in the ISM).

Figure 3 shows the comparison between the structures of a subset of the considered molecules computed at GFN2 with that resulting by the DFT optimization by Ferrero et al. The agreement is, in general, very good considering the speedup due to GFN2. Also, the critical case of the methyl radical is well reproduced.

Figure 4 compares the GFN2 BEs against the DFT ones. We exclude from the correlation these unusual cases: $\mathrm{O}_{2}, \mathrm{CO}, \mathrm{OH}, \mathrm{CH}_{3}$, simply showing the more common molecules of the set. For both the $\mathrm{BE}$ and $\mathrm{BE}(0)$ the linear correlation is close to the "ideal line", i.e. the trend line we would have obtained if the GFN2 


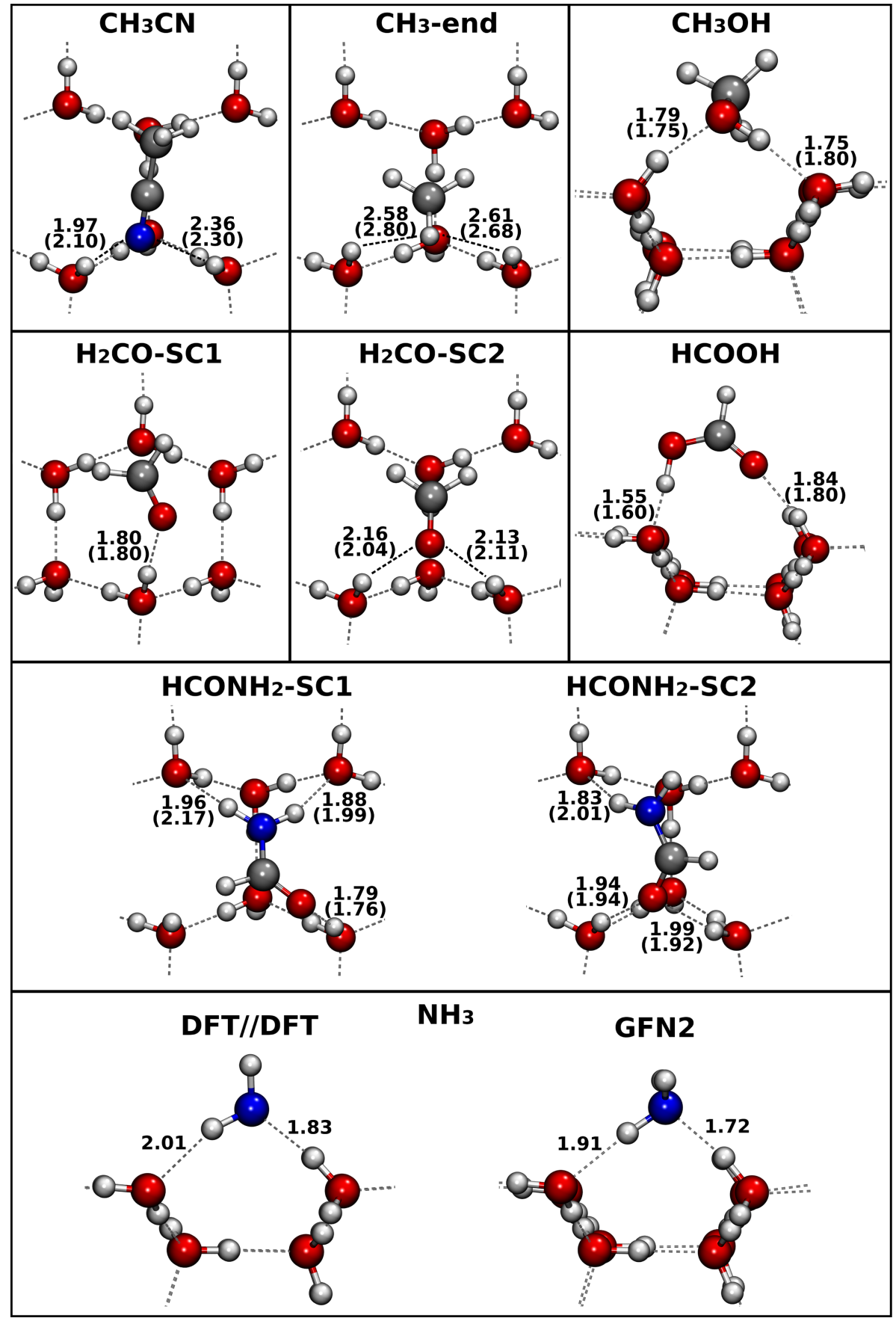

Fig. 3. Comparison between DFT structures (in parenthesis) from Ferrero et al. 2020 [8] and GFN2 results for selected molecules. Distances in unit of $\AA$. 

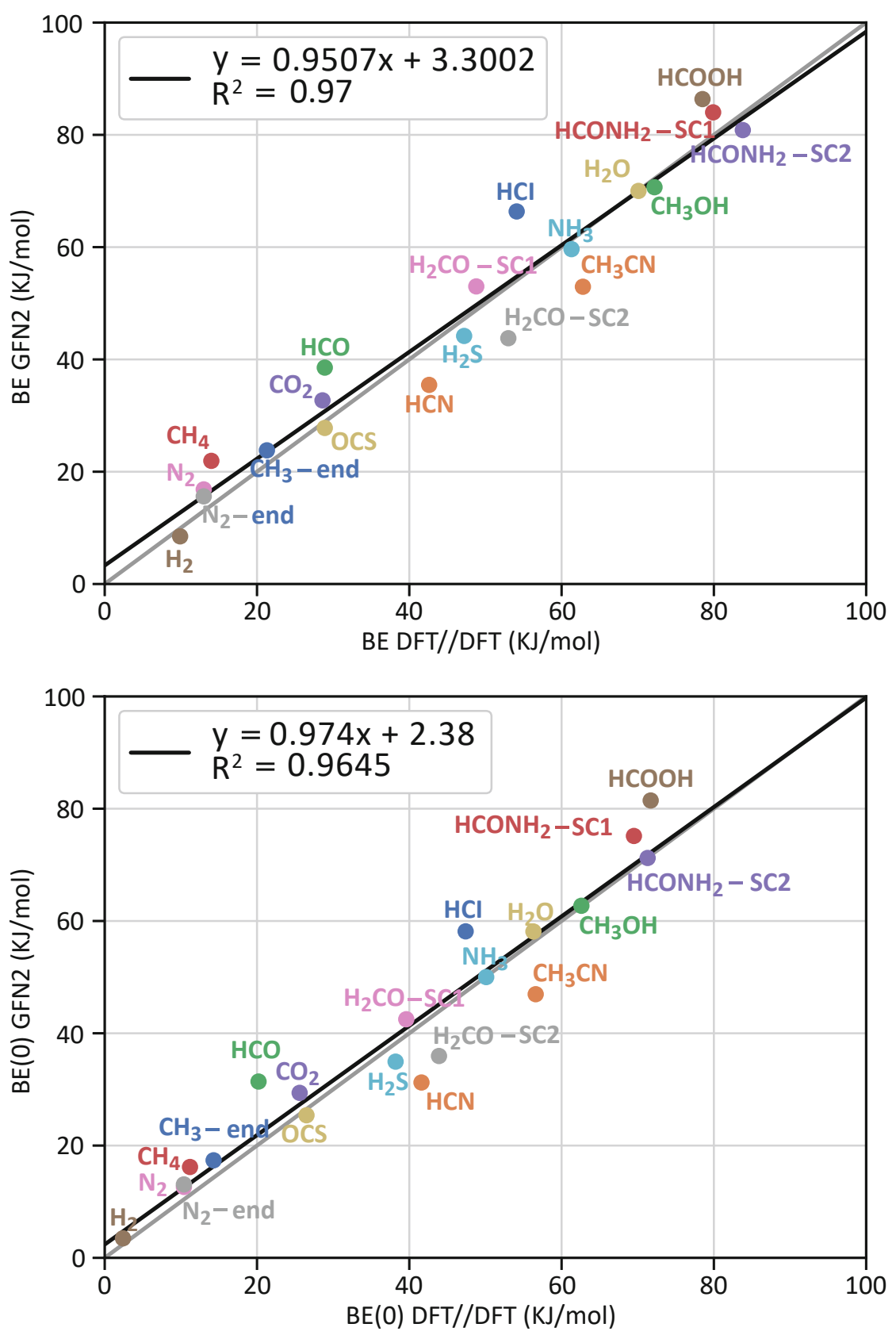

Fig. 4. Up: Linear fit (black line) between BEs using DFT//DFT results available in Ferrero et al. 2020 [8] and BEs using GFN2. The grey line represents the "ideal line". Down: Same as up except the BEs are ZPE corrected in both cases. 
results were exactly equal to the DFT ones computed by Ferrero et al. [8]. The good agreement is also evidence that the relative high dipole moment in the crystalline ice plane does not affect the GFN2 BE to a significative extent.

Fine analysis of data in Fig. 4 allows to detect specific cases with larger deviations, namely:

- HCl: $+12.2 \mathrm{~kJ} / \mathrm{mol}(22.6 \%)$ for the BE (when compared to DFT//DFT results) and $+10.8 \mathrm{~kJ} / \mathrm{mol}(22.7 \%)$ for the $\mathrm{ZPE}$ corrected $\mathrm{BE}(0)$ (when compared to ZPE corrected DFT//DFT results)

- HCN: $-7.14(16.8 \%)$ for the BE and $-10.4(24.9 \%)$ for the $\mathrm{BE}(0)$

$-\mathrm{CH}_{3} \mathrm{CN}$ : $-9.85(15.7 \%)$ for the $\mathrm{BE}$ and $-9.64(17.0 \%)$ for the $\mathrm{BE}(0)$

- $\mathrm{H}_{2} \mathrm{CO}-\mathrm{SC} 2 \mathrm{-}-9.22(17.4 \%)$ for the $\mathrm{BE}$ and $-7.95(18.1 \%)$ for the $\mathrm{BE}(0)$

- $\mathrm{HCOOH}:+7.86(10.0 \%)$ for the $\mathrm{BE}$ and $+9.76(13.6 \%)$ for the $\mathrm{BE}(0)$

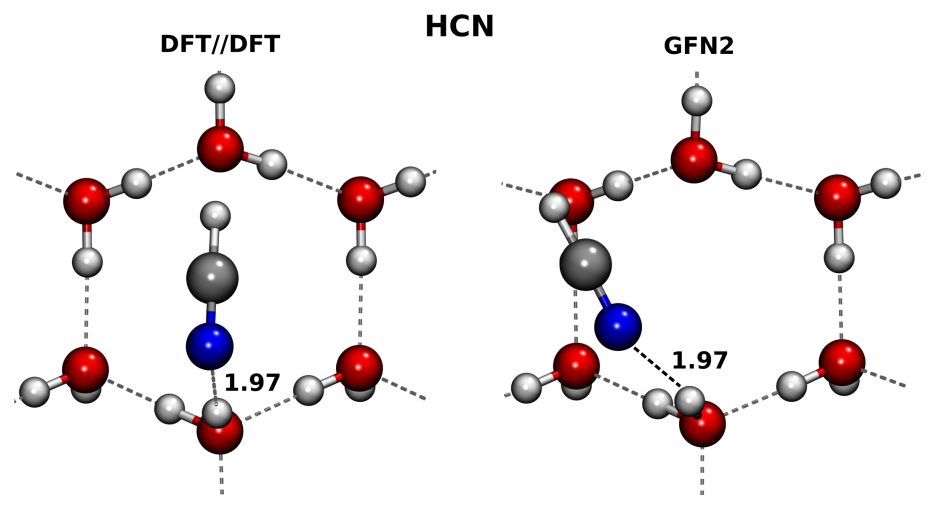

Fig. 5. Left: DFT//DFT results for HCN. From Ferrero et al. 2020 [8]. Right: GFN2 results for $\mathrm{HCN}$.

In this list, only $\mathrm{HCN}$ shows a significant difference in the final molecule position compared to Ferrero et al. 2020 [8] (as can be seen on Fig. 5). The nitrogen is still accepting the hydrogen bond from the $\mathrm{dH}$, but the HCN hydrogen does not make a H-bond with the $\mathrm{dO}$ going toward an adjacent dangling oxygen. This is due to the delicate balance between the H-bond donor/acceptor geometrical features which is handled differently by GFN2 compared to DFT. Therefore, HCN is probably a critical molecule for GFN2 for the same reasons explained for the $\mathrm{CO}$ case, as the triple bond electronic features are difficult to describe.

Correcting the $\mathrm{BE}$ for $\mathrm{ZPE}$ is important in the astrochemical context of the cold (10K) molecular clouds. Unfortunately, the frequency calculations can be quite demanding, due to the calculation of the expensive Hessian matrix. Therefore, it is important to establish how much the BE are affected by the ZPE correction. Figure 6 shows, indeed, that a very robust linear correlation exists between the two set of $\mathrm{BE}$ and $\mathrm{BE}(0)$ values. This implies that $\mathrm{BE}(0)$ values can be arrived at without actually computing the vibrational frequencies, 

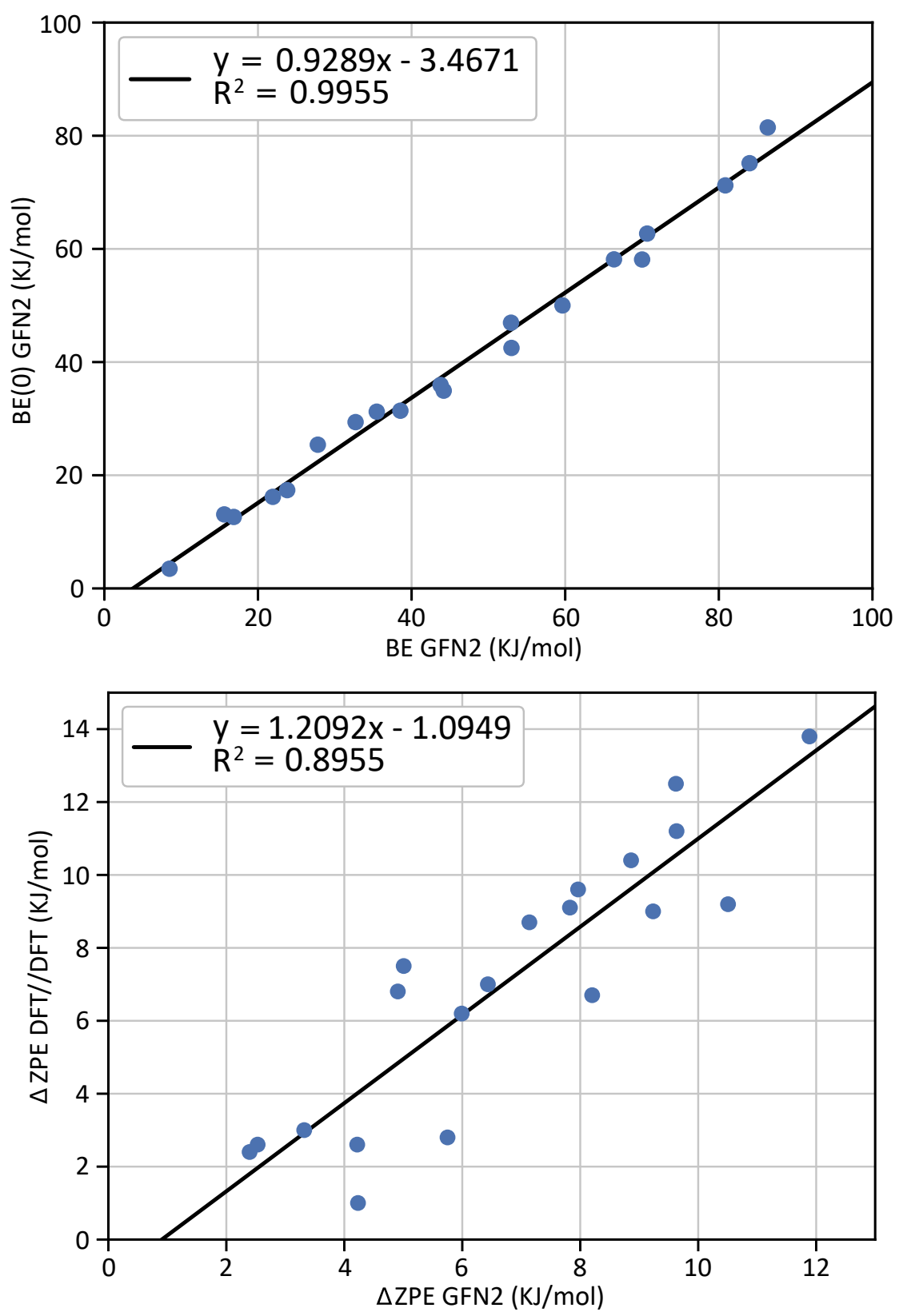

Fig. 6. Top: Linear fit between GFN2 BE and BE(0). Bottom: Linear fit between GFN2 $\triangle \mathrm{ZPE}$ and $\mathrm{DFT} / / \mathrm{DFT} \triangle \mathrm{ZPE}$. 
by a simple re-scaling of the $\mathrm{BE}$ values. This has important consequences for the future calculations in which the $\mathrm{BE}$ of $\mathrm{iCOMs}$ will be computed with respect to large amorphous icy grains than the present crystalline model. Even more important is to establish whether the $\triangle \mathrm{ZPE}$ computed by GFN2 (very cheap) correlates with the corresponding DFT ones reported by Ferrero et al. [8] (very expensive). The graph in the bottom part of Fig. 6 reveals that the correlation is only coarse: unfortunately, one cannot adopt the GFN2 $\triangle \mathrm{ZPE}$ to correct the DFT BE.

\section{Conclusion}

In this work we benchmarked the GFN2 method provided by the GFN-xTB program developed by the Grimme's group at the Bohn university, against DFT results from the work by Ferrero et al. 2020 [8]. The benchmark consisted in comparing structures and binding energies (BE) of a large set of interstellar molecules adsorbed at the surface of a crystalline, proton ordered ice cluster grain model. This cluster was cut out from the periodic P-ice adopted by Ferrero et al. [8] as a crystalline model for the interstellar ice. We also extended the comparison to study the role of zero point energy correction to the electronic BEs. The comparison is biased by the adoption of a molecular cluster of 84 water molecules to represent an otherwise periodic crystalline model as reported by Ferrero et al. 2020 [8]. Nevertheless, the results showed excellent performance of the GFN2 for both structures and $\mathrm{BE}$, with some important exceptions, namely radical species and the $\mathrm{CO}$ molecule. As many radical species are also challenging for DFT functionals, we did not expect good GFN2 performance. The bad behaviour for CO is, instead, much more serious, due to the relevance of this molecule in the ISM. Unfortunately, GFN2 badly fails in predicting even the corrected structure, in which $\mathrm{CO}$ is $\mathrm{H}$-bonded through its carbon end with the $\mathrm{dH}$ of the ice surface.

We also showed that the zero point energy corrected $\mathrm{BE}(0)$ nicely correlates with the uncorrected $\mathrm{BE}$ ones, allowing to skip the very expensive calculation of the Hessian matrix in future applications of GFN2 for the larger icy grain models.

In conclusion, we are optimistic about the application of the GFN2 method to model large icy grain models closer in size to the real grain than what has been simulated up to now. The excellent performance for structures and $\mathrm{BE}$ also allows to use GFN2 as a promising low model method in a refined ONIOM approach [5], in which high DFT/MP2/CCSD(T) level of theory for a limited portion of the icy grain where the adsorption takes place are adopted to compute very accurate $\mathrm{BE}$ values. This is the subject of future work by our group.

Acknowledgements. This project has received funding from the European Union's Horizon 2020 research and innovation programme under the Marie Skłodowska-Curie grant agreement No 811312 for the project "Astro-Chemical Origins" (ACO).

Images of 3D molecule rendering were made with VMD. VMD is developed with NIH support by the Theoretical and Computational Biophysics group at the Beckman Institute, University of Illinois at Urbana- Champaign. 


\section{References}

1. Bannwarth, C., et al.: Extended tight-binding quantum chemistry methods. WIREs Comput. Mol. Sci. 11(2), 1-49 (2021). https://doi.org/10.1002/wcms.1493

2. Bannwarth, C., Ehlert, S., Grimme, S.: GFN2-xTB - An accurate and broadly parametrized self-consistent tight-binding quantum chemical method with multipole electrostatics and density-dependent dispersion contributions. J. Chem. Theory Comput. 15(3), 1652-1671 (2019). https://doi.org/10.1021/acs.jctc.8b01176

3. Boogert, A.C., Gerakines, P.A., Whittet, D.C.: Observations of the icy universe. Ann. Rev. Astron. Astrophys. 53(1), 541-581 (2015). https://doi.org/10.1146/ annurev-astro-082214-122348

4. Casassa, S., Ugliengo, P., Pisani, C.: Proton-ordered models of ordinary ice for quantum-mechanical studies. J. Chem. Phys. 106(19), 8030-8040 (1997). https:// doi.org/10.1063/1.473813

5. Chung, L.W., et al.: The oniom method and its applications. Chem. Rev. 115(12), 5678-5796 (2015). https://doi.org/10.1021/cr5004419

6. Das, A., Sil, M., Gorai, P., Chakrabarti, S.K., Loison, J.C.: An approach to estimate the binding energy of interstellar species. arXiv 237(1), 9 (2018). https://doi.org/ $10.3847 / 1538-4365 /$ aac886

7. Enrique-Romero, J., Rimola, A., Ceccarelli, C., Ugliengo, P., Balucani, N., Skouteris, D.: Reactivity of $\mathrm{HCO}$ with $\mathrm{CH}_{3}$ and $\mathrm{NH}_{2}$ on water ice surfaces. A comprehensive accurate quantum chemistry study. ACS Earth Space Chem. 3(10), 2158-2170 (2019). https://doi.org/10.1021/acsearthspacechem.9b00156

8. Ferrero, S., Zamirri, L., Ceccarelli, C., Witzel, A., Rimola, A., Ugliengo, P.: Binding energies of interstellar molecules on crystalline and amorphous models of water ice by ab-initio calculations. Astrophys. J. 904(1), 11 (2020). https://doi.org/10.3847/ 1538-4357/abb953

9. Frenking, G., Loschen, C., Krapp, A., Fau, S., Strauss, S.H.: Electronic structure of CO-an exercise in modern chemical bonding theory. J. Comput. Chem. 28(1), 117-126 (2007). https://doi.org/10.1002/jcc.20477

10. Germain, A., Ugliengo, P.: Modeling interstellar amorphous solid water grains by tight-binding based methods: comparison between GFN-XTB and CCSD(T) results for water clusters. In: Gervasi, O., et al. (eds.) ICCSA 2020. LNCS, vol. 12253, pp. 745-753. Springer, Cham (2020). https://doi.org/10.1007/978-3-03058814-4_62

11. Muenter, J.: Electric dipole moment of carbon monoxide. J. Mol. Spectrosc. 55(13), 490-491 (1975). https://doi.org/10.1016/0022-2852(75)90287-8

12. Oba, Y., Miyauchi, N., Hidaka, H., Chigai, T., Watanabe, N., Kouchi, A.: Formation of compact amorphous $\mathrm{H}_{2} \mathrm{O}$ ice by codeposition of hydrogen atoms with oxygen molecules on grain surfaces. Astrophys. J. 701(1), 464-470 (2009). https:// doi.org/10.1088/0004-637X/701/1/464

13. Pantaleone, S., Enrique-Romero, J., Ceccarelli, C., Ugliengo, P., Balucani, N., Rimola, A.: Chemical desorption versus energy dissipation: insights from ab-initio molecular dynamics of HCO formation. arXiv 897(1), 56 (2020). https://doi.org/ $10.3847 / 1538-4357 / \mathrm{ab} 8 \mathrm{a} 4 \mathrm{~b}$

14. Rimola, A., et al.: Can formamide be formed on interstellar ice? An atomistic perspective. ACS Earth Space Chem. 2(7), 720-734 (2018). https://doi.org/10. 1021/acsearthspacechem.7b00156

15. Vidali, G.: $\mathrm{H}_{2}$ formation on interstellar grains. Chem. Rev. 113(12), 8762-8782 (2013). https://doi.org/10.1021/cr400156b 
16. Wakelam, V., Loison, J.C., Mereau, R., Ruaud, M.: Binding energies: new values and impact on the efficiency of chemical desorption. Mol. Astrophys. 6, 22-35 (2017). https://doi.org/10.1016/j.molap.2017.01.002

17. Wakelam, V., et al.: $\mathrm{H}_{2}$ formation on interstellar dust grains: the viewpoints of theory, experiments, models and observations. Mol. Astrophys. 9, 1-36 (2017). https://doi.org/10.1016/j.molap.2017.11.001

18. Watanabe, N., Kouchi, A.: Ice surface reactions: a key to chemical evolution in space. Prog. Surf. Sci. 83(10-12), 439-489 (2008). https://doi.org/10.1016/j. progsurf.2008.10.001

Open Access This chapter is licensed under the terms of the Creative Commons Attribution 4.0 International License (http://creativecommons.org/licenses/by/4.0/), which permits use, sharing, adaptation, distribution and reproduction in any medium or format, as long as you give appropriate credit to the original author(s) and the source, provide a link to the Creative Commons licence and indicate if changes were made.

The images or other third party material in this chapter are included in the chapter's Creative Commons licence, unless indicated otherwise in a credit line to the material. If material is not included in the chapter's Creative Commons licence and your intended use is not permitted by statutory regulation or exceeds the permitted use, you will need to obtain permission directly from the copyright holder.

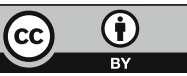

